\title{
Fosfojips Katkısının Duvar Karosu Opak Sır Fiziksel Özelliklerine Etkisi
} The Effect of Phosphogypsum Addition on Physical Properties of Wall Tile Opaque Glaze

\author{
Lina İSRAEL İSRAIL ${ }^{* a}$, Kemal KÖSEOĞLU ${ }^{b}$
}

Ege Üniversitesi, Ege Meslek Yüksekokulu, Seramik Cam ve Çinicilik Programı, 35100, İzmir

• Geliş tarihi / Received: 14.05.2019 • Düzeltilerek geliş tarihi / Received in revised form: $24.10 .2019 \quad$ • Kabul tarihi / Accepted: 28.10 .2019

\section{$\ddot{O} z$}

Bu çalışmada, duvar karosu opak sır (DKOS) reçetesine farklı oranlarda katılan fosfojips (FJ) in pişirme öncesi seramik çamurdaki etkileri viskozite, tiksotropi ve yoğunluk testleri incelenmiştir. Daldırma yöntemiyle hazırlanmış sırlı karolar $950,1050,1150^{\circ} \mathrm{C}$ de ayrı ayrı pişirilmiștir. Çarpmaya, 1sıl şoka, kimyasallara dayanım ve renk testlerine tabi tutularak fiziksel özellikleri araştırılmıştır. DKOS1 örneği ile opak sırın akıcıllı̆ 1 artmış ve $1150^{\circ} \mathrm{C}$ pişirim sonrası tüm mekanik testler olumlu sonuçlanmıştır. FJ in sır reçetesine katılmasının geri dönüştürülmesi açısından uygun bir yöntem olduğu görülmüş̧ür.

Anahtar kelimeler: Fosfojips, Mekanik Testler, Opak Sır, Sinterleşme

\begin{abstract}
The effects of addition of phosphogypsum to wall tile opaque glaze receipe are investigated by viscosity, thixpthropy and density tests before sintering. Tiles which are prepared by dipping method are sintered seperately at $950^{\circ}, 1050^{\circ}$ and $1150^{\circ} \mathrm{C}$ anda re subjected to impact resistence, thermal shock, resistance to chemicals and color tests for searching physical properties of the tiles. The flow behaviour of opaque glaze increased with DKOS1 sample and all mechanical tests gave positive results after firing at $1150^{\circ} \mathrm{C}$. The addition of phosphogypsum is considered as an appropriate method for recycling phosphogypsum.
\end{abstract}

Keywords: Phosphogypsum, Mechanical Tets, Opaque Glaze, Sintering

\footnotetext{
$*^{\mathrm{a}}$ Lina İSRAEL İSRAİL; lina.israil.01@ mail.ege.edu.tr, Tel: (0232) 311 29 39, orcid.org/0000-0002-0560-1839

${ }^{\mathrm{b}}$ orcid.org/0000-0003-1116-9103
} 


\section{Giriş}

Sirlama, seramik bünyeleri ince, sert cam tabaka ile kaplamadır. Bu cam tabaka vitröz faz, heterojen fazlar veya \%80-90 oranında cam matriksten oluşmuş cam-seramik fazları içerir (da Silva vd, 2012). Yüzeyin gözenekliliğini azaltarak bünyeye mekanik mukavemet, elektriksel yalıtkanlık, kimyasal maddelere karşı direnç ve estetik özellikler kazandırır. Çeşitli atıkların reçete içine katılması ve sır üretiminde kullanılması malzemelerin geri dönüşümü için iyi bir seçenektir.

Farklı sanayi dallarının yan ürün ve atık olarak nitelendirdiği çeşitli ürünlerin kullanımı, ekonomiye kazandırılması; doğal ve ithal edilen hammadde tüketiminin, büyük yığınlar halinde depolanan ürün miktarının ve dolayısı ile çevre kirliliği problemlerinin azalmasına yardımcı olur (Sirazhiddinov vd.,1994).

Dünya nüfusunun II. Dünya Savaşından bu yana hızlı artışı sonucu, gübre kullanımı açlık ve kıtlığa karşı mücadelede kısmi bir sonuç olmuştur. Gübre hammaddelerinden biri olan fosfatın fosfat kayalarından elde edilmesi ve fosforik asit üretimi için kullanılması oldukça önemlidir (URL 1, 2007). Fosfat kayaçları ile sülfürik asidin tepkimesi sonucu fosforik asit ve fosfat gübreleri yanında, alçıtaşı ile aynı kimyasal yapıya sahip FJ yan ürün olarak oluşur (Reaksiyon 1) (Gennari R.F. vd., 2011)

$\mathrm{Ca}_{10}\left(\mathrm{PO}_{4}\right)_{6} \mathrm{~F}_{2}+10 \mathrm{H}_{2} \mathrm{SO}_{4}+20 \mathrm{H}_{2} \mathrm{O} \longrightarrow$

$10 \mathrm{CaSO}_{4} \cdot 2 \mathrm{H}_{2} \mathrm{O}+6 \mathrm{H}_{3} \mathrm{PO}_{4}+2 \mathrm{HF}$

Fosfojips (FJ)

ve $\mathrm{P}_{2} \mathrm{O}_{5}$, florür iyonları ve organik maddeler gibi safsızlıklar içerir (Smadi vd., 1999; Jonker ve Potgieter, 2005). Yaş yöntem olarak adlandırılan bu üretim şeklinde (Reaksiyon 1) fosforik asit ve FJ süzülerek birbirinden ayrılır (Demirel ve Çağlar, 2015). Devlet Planlama Teşkilatının 2001 y1lı Madencilik Özel İhtisas Komisyon Raporuna göre Türkiye' de her yıl yaklaşık 3 milyon ton atık FJ açığa çıkmakta (URL 2, 2007) ve fabrikalarda açıkta depolanmaktadır (Demirel ve Çağlar, 2015). Bu rakam dünya genelinde 280 milyon tondur (Zhou vd., 2016). Üretimi ile hava ve deniz kirliliğine neden olan, depolanması ile kara kirliliğine de sebebiyet veren $\mathrm{FJ}$ için uygun depolama alanlarının oluşturulması üretim maliyetini de arttırır (Demirel ve Çağlar, 2015). Uygun yöntemlerle bu kirliliğin azaltılmas1 çalışmaları yapılmalı ve geri kazanım yolları araştırılmalıdır.
FJ inşaat ve yap1 malzemeleri sektöründe alçı panel yapımında (Garg vd, 2011; Zhou vd., 2016), pişirilmemiş tuğla (Değirmenci 2008; Zhou vd., 2012, 2016), siva (Singh, 2002) yapımında kullanılması ve özellikle Portland çimentosu için donmayı geciktirici olarak doğal alçıtaşı yerine kullanılması önerilmiştir. (Akın ve Sert, 2004; ElDidamony vd, 2013; Zhou vd., 2016). Tarımda toprağın $\mathrm{pH}$ ve kalsiyum miktarını ayarlayıcı olarak (Değirmenci vd, 2007; Zhou vd., 2016), kimya sektöründe çimento üretimi için kullanımı araştırılmıştır (Garg vd, 2011; Zhou vd., 2016). Seramik sektöründe bağlayıcı olarak fosfat çimentosunda ve doymamış polimer reçinesi şeklinde kullanımı önerilmiştir (Formosa vd, 2012; Li vd.,2013; Wang vd, 2013; Zhou vd., 2016).

$\mathrm{Bu}$ çalışmanın amacı kimyasal atık olan FJ in, duvar karosu opak sira (DKOS) katılması ile hazırlanan duvar karolarının fiziksel ve mekanik dayanıklılığını faklı pişirme sıcaklıklarında standartlara uygun olarak araştırmaktır. Özellikle FJ in sır üretiminde hiç kullanılmamış olması önemlidir.

\section{Malzeme ve Yöntem}

\subsection{Sirlı Duvar Karolarının Hazırlanması}

DKOS, Ege Seramik Fabrikasından (Polat Holding A.Ş, İzmir), FJ ise Toros Tarım Sanayi ve Ticaret Anonim şirketinden temin edilmiştir. Tablo 1 ve Tablo 2'de sirasiyla DKOS kimyasal bileşimi ve Seger formülü, Tablo 3'de ise FJ kimyasal bileşimi verilmiştir.

Öğütülmüş ve etüvde kurutulmuş DKOS, numune kabına yerleştirildikten $10^{\circ} \mathrm{C} / \mathrm{dk}$ 1sıtma hızı ile $400-1200^{\circ} \mathrm{C}$ sıcaklık aralığında çalışan 1s1 mikroskobunda (Misura 3.32) 1sıl davranış1 incelenmiştir (Şekil 1). Sir $876^{\circ} \mathrm{C}$ de sinterleşmiş, $1200^{\circ} \mathrm{C}$ den sonra akış gerçekleşmiştir.

Ağırlıkça 1, 2.5, 5, 7.5 10, 15 yüzde bileşimlerinde DKOS na ayrı ayrı katılan FJ örnekleri (Tablo 4) manyetik karıştırıcıda (Chiltern) 1 saat karıştırılarak homojen süspansiyonlar elde edilmiştir. $5 \mathrm{~cm} \times 5 \mathrm{~cm} \times 0.5 \mathrm{~cm}$ ebatlarında kesilmiş duvar karolar $105^{\circ} \mathrm{C}$ de etüvde (Heraus) 2 saat kurutulup oda sicaklığına soğutulmuştur. Süspansiyonlar karolara daldırma yöntemi ile uygulanmıştır. Etüvde 2 saat bekletilen numuneler $25^{\circ} \mathrm{C} / \mathrm{dk}$ 1sıtma hızı ile üç farklı pişirme sicaklığında $\left(950, \quad 1050, \quad 1150 \quad{ }^{\circ} \mathrm{C}\right) \quad 2$ saat pişirilmiştir (Protherm). 
Tablo 1. DKOS kimyasal bileşimi

\begin{tabular}{c|cccccccc}
\hline Oksit ad1 & $\mathrm{SiO}_{2}$ & $\mathrm{Al}_{2} \mathrm{O}_{3}$ & $\mathrm{CaO}$ & $\mathrm{MgO}$ & $\mathrm{Na}_{2} \mathrm{O}$ & $\mathrm{K}_{2} \mathrm{O}$ & $\mathrm{ZnO}$ & $\mathrm{BaO}$ \\
\hline$\%$ & 58.21 & 11.26 & 11.35 & 1.11 & 5.76 & 2.84 & 8.42 & 1.05 \\
\hline
\end{tabular}

Tablo 2. DKOS Seger formülü
$0.2 \mathrm{Na}_{2} \mathrm{O}$
$0.07 \mathrm{~K}_{2} \mathrm{O}$
$0.22 \mathrm{ZnO}$
$0.02 \mathrm{BaO}$
$0.42 \mathrm{CaO}$
$0.07 \mathrm{MgO}$

$0.24 \mathrm{Al}_{2} \mathrm{O}_{3}$

$2.11 \mathrm{SiO}_{2}$

Tablo 3. FJ kimyasal bileşimi

\begin{tabular}{l|lllllll}
\hline Hammadde ad1 & $\mathrm{KK}$ & $\mathrm{SiO}_{2}$ & $\mathrm{Al}_{2} \mathrm{O}_{3}$ & $\mathrm{Fe}_{2} \mathrm{O}_{3}$ & $\mathrm{CaO}$ & $\mathrm{SO}_{3}$ & $\mathrm{P}_{2} \mathrm{O}_{5}$ \\
\hline FJ (Eritme-\%) & 19.98 & 1.54 & 1.23 & 0.04 & 44.13 & 31,95 & 1.1 \\
FJ (Kat1 pres-\%) & - & 1.22 & 0.43 & 0.02 & 34.25 & 63.08 & 1 \\
\hline
\end{tabular}

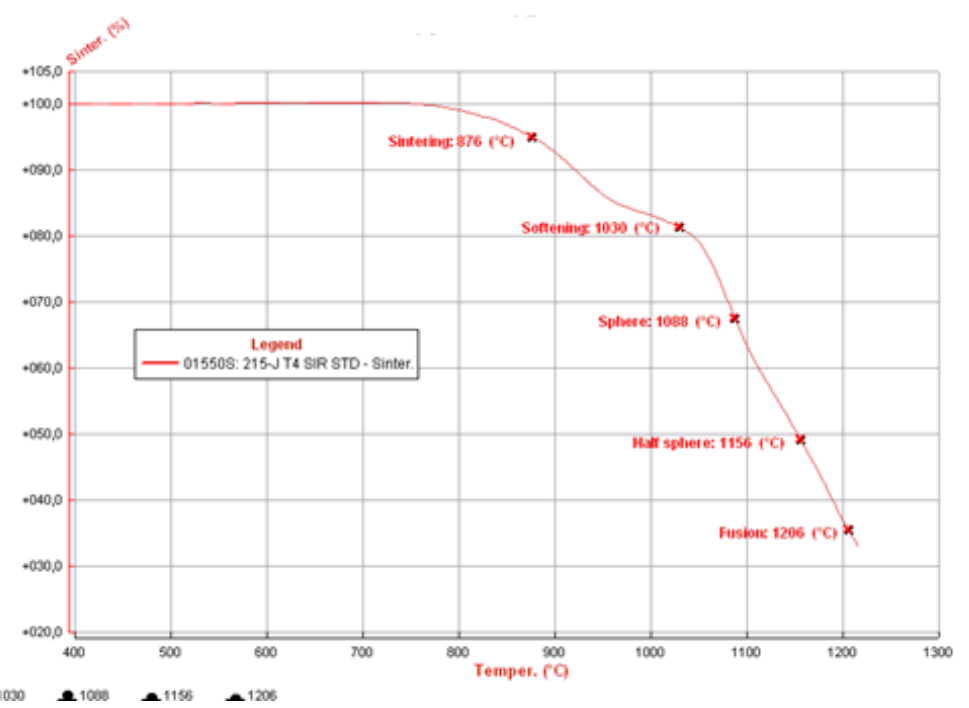

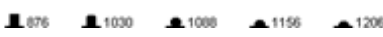

Şekil 1. DKOS erime davranış1

Tablo 4. FJ katkılı DKOS örneklerinin deneysel gösterimi

\begin{tabular}{l|l}
\hline FJ bileşimi (\%) & Gösterim şekli \\
\hline 0 & DKOS \\
1 & DKOS 1 \\
2.5 & DKOS 2.5 \\
5 & DKOS 5 \\
7.5 & DKOS7.5 \\
10 & DKOS10 \\
15 & DKOS15 \\
\hline
\end{tabular}

Pişme öncesi Tablo 4'de verilen süspansiyon örneklerinin viskozite, tiksotropi ve yoğunluk testleri, yapılmıştır. Bu süspansiyonların karolara uygulanıp pişirilmelerinden sonra ise çarpmaya direnç testi, 1s1 şokuna dayanım testi, kimyasallara karşı dayanıklılık testi ve renk testleri ilgili standartlara uygun şekilde yapılmıştır.

\subsection{Viskozite ve Tiksotropi Testleri}

Şekil 2'de viskozite ve tiksotropi denemelerinde kullanılan, alt delik çapı $4 \mathrm{~mm}$ olan fordcup gösterilmektedir. DIN 53211 standardına gereği ortalama akış süreleri $\left(t_{1}\right)$ fordcup ile belirlenen süspansiyonların kinematik viskoziteleri dönüşüm tabloları (URL 3) kullanılarak hesaplanmıştır.

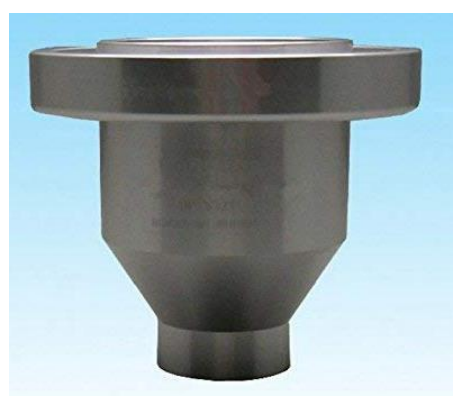

Şekil 2. Viskozite ve tiksotropi denemelerinde kullanılan fordcup 
Zamana bağlı olarak akıcılık değişimi olarak tanımlanan tiksotropi denemelerinde her örneğin akış süresine, fordcup içinde 10 dakika bekletildikten sonra bakilır $\left(\mathrm{t}_{2}\right)$.

$\%$ Tiksotropi $=\frac{\mathrm{t}_{2-} \mathrm{t}_{1}}{\mathrm{t}_{2}} \times 100$

formülü ile hesaplanır.

\subsection{YığınYoğunluğu Testi}

Yoğunluk tayini TS EN ISO 10545-3 standardına göre yapilmıştır. $20^{\circ} \mathrm{C}$ de saf su ile net hacmi tayin edilen $25 \mathrm{~mL}$ lik piknometrelerin içersine dökülen süspansiyonların tartımı alınır ve süspansiyon kütlesi hesaplanır. $\mathrm{Bu}$ değer piknometre hacmine bölünerek yığın yoğunluğu değeri bulunur.

\section{4 Çarpmaya Direnç Testi}

TSE EN ISO 10545-5 standard 1 gereği $5 \mathrm{~cm}$ x 5 $\mathrm{cm}$ x $0.5 \mathrm{~cm}$ ebatlarındaki karoların üzerine $50 \mathrm{~cm}$ yükseklikten bırakılan çelik topun $(50 \pm 2 \mathrm{~g})$ zemin üzerinde çatlak oluşumuna sebebiyet verip vermediği kontrol edilir.

\subsection{Isıl Şok Dayanım Testi}

Karolar etüvde (Heraus) $120^{\circ} \mathrm{C}$ de 20 dakika bekletilir. Sürenin sonunda sıcaklığ $20^{\circ} \mathrm{C}$ olan su dolu kaba yerleştirilir. 10 dakika bekletilir. Kaptan alınan karolar kuru temiz bir bez yardımı ile silindikten sonra zemin üzerinde çatlak oluşumu kontrol edilir. Aynı işlem $150^{\circ}, 160^{\circ} \mathrm{C}, 180^{\circ}$ ve $200^{\circ} \mathrm{C}$ için tekrarlanır (TS EN ISO 10545-9). $200^{\circ} \mathrm{C}$ de çatlamayan karoların sürekli kullanılabileceği Tablo 5'den görülmektedir.

\subsection{Kimyasallara Dayanıklılı Testi}

Pişmiş karoların asit ve alkalilere dayanıklılık testi TS EN ISO 10545-13 standardina uygun yapılmıştır. Yüzeyleri alkol ile temizlenen karolar içinde $250 \mathrm{~mL}$ hacimce $\% 18 \mathrm{HCl}$ çözeltisi içeren ve hacimce $\% 10 \mathrm{KOH}$ içeren kaplara ayrı ayrı yerleştirilir. Kaplar oda sıcaklığında dört gün bekletilir. Sürenin bitiminde çıkarılan karoların yüzeyleri su ile yıkanır ve alkolle ile temizlenir. Karoların yüzeyinde matlaşma olup olmadığ 1 kontrol edilir.

Tablo 5. Sirlı numunelerde sicaklık ve çatlağa karşı dayanma süresi arasındaki ilişki (Parmelee, 1987 Yalçın ve Sevinç, 2000).

\begin{tabular}{cccccc}
\hline Sicaklık $\left({ }^{\circ} \mathrm{C}\right)$ & 120 & 150 & 160 & 180 & 200 \\
\hline Kullanım & 8 & $3-4$ & 15 ay & $2-3$ & Sürekli \\
süresi & gün & ay & & yıl & \\
\hline
\end{tabular}

\subsection{Renk Test}

Opaklık derecesi Euromeccanica X-rite trikromatik renk ölçüm cihazı kullanarak TS EN ISO 10545-16 standardı ile uyumlu olarak gerçekleştirilmiştir. Opaklık derecesi L değeriyle, yeşil-kırmızı renk aralığı a değeriyle, mavi-sarı renk aralığ $\mathrm{b}$ değeriyle verilmektedir. Standart ve numuneler arasındaki renk farkı, $\square \mathrm{E}$, uluslararası renk ölçüm sistemine göre (CIELAB sistemi) hesaplanmıştır.

$\Delta \mathrm{E}=\sqrt{\left(\mathrm{L}_{1}-\mathrm{L}_{2}\right)^{2}+\left(\mathrm{a}_{1}-\mathrm{a}_{2}\right)^{2}+\left(\mathrm{b}_{1}-\mathrm{b}_{2}\right)^{2}}$

1 ve 2 indisleri sirasiyla numune ve standard temsil etmektedir (URL 5).

\section{Bulgular}

\subsection{Viskozite ve Tiksotropi Testleri}

Şekil 3 FJ katk1lı DKOS örneklerinin kinematik viskozitelerini göstermektedir.



Şekil 3. FJ katkısı ile DKOS in kinematik viskozite değişimi

Standart DKOS in akış süresi $15 \mathrm{~s}$ dir. Bu süre DKOS1 örneği ile 12 saniyeye düşmüş, akıcılık artmıştır. Bu noktadan sonra FJ katkı oranının artmasıyla akış süresi de yavaş yavaş artmış ve DKOS15 numunesinin akış süresi 20 saniyenin üzerine çıkmıştır. Yer yer oluşan floklaşmalar durumu olumsuz kılmış, akışı zorlaştırmıştır. Tiksotropi değerlerinin artan FJ derişimleri ile değişimi Şekil 4'de verilmiştir. Standart DKOS in tiksotropi yüzdesi 1 dir. $\mathrm{Bu}$ değer, DKOS1 örneğinde $\% 0.97$ değerine düşmüş ardından kademeli olarak artmış ve DKOS15 sirlı örneğinde \% 1.7 değerine çıkmıştır. Akışın bu noktada zorlaştığ 1 yüksek tiksotropi değerinden de anlaşılmaktadır. Sadece ağırlıkça yüzde 1 FJ katkısı çamuru daha akışkan hale getirmiştir. 


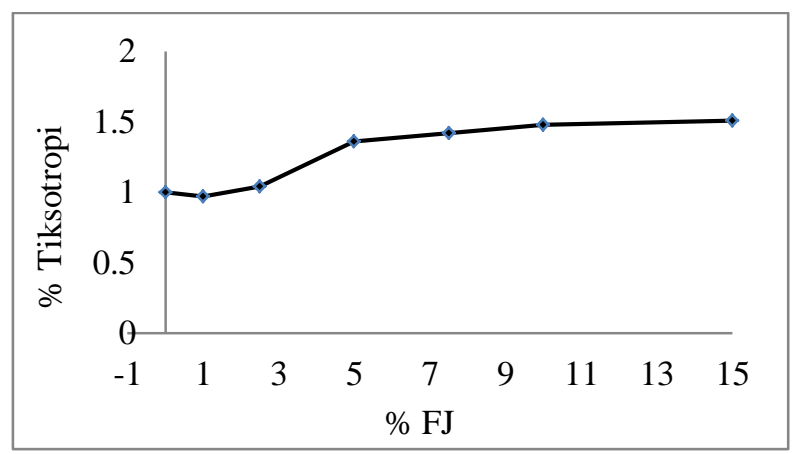

Şekil 4. FJ katkısı ile DKOS in tiksotropi değişimi

FJ $\left(\mathrm{CaSO}_{4} \cdot 2 \mathrm{H}_{2} \mathrm{O}\right)$ in sinterleşmesinde açığa çıkan $\mathrm{CaO}$ iyi bilinen eritici toprak alkali oksittir. Eriticilerin aşırı kullanılması sır yapısında bağ yapmamış oksijenlerin sayısını arttırarak yapıyı zayıflatır. $\mathrm{Bu}$ durum özellikle sırın cam özelliklerini olumsuz etkiler (Mejia, 2004). Erimiş sır yapısında oluşan kalsiyum silikatın sınırlı çözünürlüğü olduğu için yapıda kristalleşmeler olur. Böyle bir olumsuzluk akıcıllğa engeldir. $\mathrm{CaO}$ miktarının giderek artmasıyla akışın zorlaştığı Şekil 3 ve Şekil 4'den açıça görülmektedir.

\subsection{Yoğunluk Tayini}

Daldırma sırlarında yoğunluk $1.5 \mathrm{~g} / \mathrm{mL}$ ile 1.7 $\mathrm{g} / \mathrm{mL}$ arasindadır (URL 6). Standart DKOS in yoğunluğu $1.42 \mathrm{~g} / \mathrm{mL}$ dir. DKOS1 örneğinin yoğunluğu $1.39 \mathrm{~g} / \mathrm{mL}$ değerine düşmüştür. Akıcılığın artması yoğunluk değerini de düşürmüştür. $\mathrm{Bu}$ noktadan sonra, artan $\mathrm{FJ}$ miktarı yoğunluğu da arttırmıştır. DKOS15 çamurunun yoğunluğu $1.7 \mathrm{~g} / \mathrm{mL}$ olarak ölçülmüştür. Sır kalınlığ $\operatorname{artmıştır~(Şekil~5).~}$



Şekil 5. FJ katkısı ile DKOS in yoğunluk değişimi

\section{3 Çarpmaya Dayanıklılık Testi}

TS EN ISO 10545-5 standardına uygun olarak yapılan bu test sonucu pişirilmiş numunelerde çarpma sonrası kırılma olmamıştır. Bu durum $\mathrm{CaO}$ katkısının sırın mekanik mukavemetini ve gerilme gücünü artırma özelliği ile açılanabilir (Lin ve Luo, 2005). Ayrica $950^{\circ} \mathrm{C}$ 'de pişirilen DKOS1 ve DKOS3 numunelerinde küçük çatlaklar gözlenmiştir (Şekil 6).



Şekil 6. Çarpmaya dayanıklılık testi uygulanmış bazı karo örnekleri

\subsection{Isıl Şoka Dayanım Testi}

Sırın genleşme katsayısı, bünyeninkinden fazla olması durumunda sır tabakasının zayıf çekme geriliminden dolayı sır yüzeyinde çatlak oluşumuna sebebiyet verir (Parmelee, 1987; Yalçın ve Sevinç, 2000). Sir yüzeyinin termal genleşme katsayısını düşürmek için belli sınırlar aralığında bünyenin $\mathrm{SiO}_{2}$ yüzdesi arttırılır, eritici oksit yüzdesi düşürülür (Singer ve Singer, 1979).

$950^{\circ} \mathrm{C}$ de pişirilen DKOS1 ve DKOS3 örnekleri $150^{\circ} \mathrm{C}$ 'de, DKOS5 ve DKOS7.5 örnekleri $180^{\circ}{ }^{\prime} \mathrm{C}$ de çatlamıştır. DKOS10 ve DKOS15 örneklerinde çatlama olmamıştır. $1050^{\circ} \mathrm{C}$ de pişirilen örneklerden sadece DKOS1 ve DKOS3 $170^{\circ} \mathrm{C}$ de çatlamıştır. Diğer örneklerde çatlama olmamıştır. $1150^{\circ} \mathrm{C}$ de hiçbir örnek çatlamamıştır.

\subsection{Kimyasallara Dayanım Testi}

$\mathrm{BaO}, \mathrm{CaO}$ veya $\mathrm{MgO}$ gibi toprak alkali oksitlerin sır içinde varlığı, sırı asit ve/veya alkali çözeltilere karşı daha az dirençli olmasına (Muller ve Zamek, 2011) ve matlaşmaya sebebiyet verir. Bu duruma $\mathrm{CaO}$ katkılı tüm pişmiş örneklerde rastlanmıştır.

\subsection{Renk Testi}

Tablo 6 standart ve FJ içeren DKOS örneklerinin trikromatik renk değerlerini göstermektedir. $\Delta \mathrm{E}$, renk farkı değerlerinin 1 den küçük olması standart ve örneklerin uyumlu olduğunu göstermektedir. 
Tablo 6. DKOS ve FJ katkı1ı örneklerin farklı pişirme sıcaklıklarındaki trikromatik renk değerleri

\begin{tabular}{c|cccccccc}
\hline $\mathbf{T}\left({ }^{\circ} \mathbf{C}\right)$ & \multicolumn{7}{c}{$\mathbf{9 5 0}$} \\
\hline $\mathbf{D K O S}$ & $\mathrm{L}$ & $\mathrm{a}$ & $\mathrm{b}$ & $\Delta \mathrm{L}^{2}$ & $\Delta \mathrm{a}^{2}$ & $\Delta \mathrm{b}^{2}$ & $\Sigma$ & $\Delta \Sigma$ \\
\hline $\mathbf{0}$ & 92.57 & 1.43 & 5.38 & & & & & \\
$\mathbf{1}$ & 91.88 & 2.08 & 5.69 & 0.48 & 0.42 & 0.1 & 0.99 & 0.50 \\
$\mathbf{2 . 5}$ & 91.75 & 2.2 & 5.8 & 0.67 & 0.59 & 0.18 & 1.44 & 0.72 \\
$\mathbf{5}$ & 92.78 & 0.73 & 5.01 & 0.04 & 0.49 & 0.14 & 0.67 & 0.34 \\
$\mathbf{7 . 5}$ & 92.14 & 0.76 & 4.96 & 0.19 & 0.45 & 0.18 & 0.81 & 0.41 \\
$\mathbf{1 0}$ & 92.36 & 0.75 & 5.37 & 0.04 & 0.46 & 0 & 0.51 & 0.25 \\
$\mathbf{1 5}$ & 92.48 & 2.08 & 5.46 & 0.01 & 0.42 & 0.01 & 0.44 & 0.22 \\
\hline & & & & $\mathbf{1 0 5 0}$ & & & & \\
\hline $\mathbf{0}$ & 92.00 & 1.77 & 7.23 & & & & & \\
$\mathbf{1}$ & 91.70 & 2.16 & 7.99 & 0.1 & 0.15 & 0.58 & 0.83 & 0.42 \\
$\mathbf{2 . 5}$ & 91.40 & 2.41 & 8.29 & 0.42 & 0.41 & 1.12 & 1.96 & 0.98 \\
$\mathbf{5}$ & 91.20 & 2.43 & 7.26 & 0.64 & 0.44 & 0 & 1.08 & 0.54 \\
$\mathbf{7 . 5}$ & 92,30 & 1.17 & 7.46 & 0,07 & 0.36 & 0.05 & 0.48 & 0.24 \\
$\mathbf{1 0}$ & 92,10 & 1.14 & 7.45 & 0 & 0.4 & 0.05 & 0.45 & 0.22 \\
$\mathbf{1 5}$ & 91,40 & 2.32 & 7.93 & 0.34 & 0.3 & 0.49 & 1.13 & 0.56 \\
\hline & & & & $\mathbf{1 1 5 0}$ & & & & \\
\hline $\mathbf{0}$ & 92.88 & 0.58 & 8.50 & & & & & \\
$\mathbf{1}$ & 92.65 & 0.2 & 8.12 & 0.05 & 0.14 & 0.14 & 0.34 & 0.17 \\
$\mathbf{2 . 5}$ & 93.76 & 1.56 & 8.84 & 0.77 & 0.96 & 0.12 & 1.85 & 0.93 \\
$\mathbf{5}$ & 92.04 & 0.26 & 8.58 & 0.71 & 0.10 & 0.85 & 1.65 & 0.83 \\
$\mathbf{7 . 5}$ & 92.65 & -0.17 & 8.16 & 0.05 & 0.56 & 0.12 & 0.73 & 0.37 \\
$\mathbf{1 0}$ & 92.48 & -0.19 & 8.56 & 0.16 & 0.59 & 0.88 & 1.64 & 0.82 \\
$\mathbf{1 5}$ & 92.7 & -0.14 & 8.76 & 0.03 & 0.52 & 0.55 & 1.10 & 0.55 \\
\hline & & & & & & & &
\end{tabular}

L değeri (opaklık, beyazlık) pişme sıcaklı̆̆ının artışı ile değişmezken, $b$ değeri artmıştır. Bu durum karolarda sar1 tonunun daha belirginleștiğini göstermektedir. a değerlerinin $1150^{\circ} \mathrm{C}$ de negatif değerlere düşmüş olması açık yeşil rengi ifade eder.

\section{Sonuçlar}

$\mathrm{Bu}$ çalışmada opak sıra ilave edilen FJ in ağırlıkça $\% 1$ oranında reçetede yer alması sırın viskozite ve tiksotropi değerlerini düşürmüş ve sıra akıcılık kazandırmıştır. $1150^{\circ} \mathrm{C}$ de pişirim sonrası yapılan mekanik testler sonucu tüm katkılı karolarda kırılma ve çatlama olmazken, opasite korunmuş ancak yüzeyde matlaşma görülmüştür. Yapılan testler sonucunda ağırlıkça \%1 FJ katkılı pişmiş sırlı duvar karosu numunesinin standart değere uygun olduğu sonucuna varılmıştır.

\section{Kaynaklar}

Akın, I.A. ve Sert, Y., 2004. Utilization weathered phosphogypsum as set retarder in portland cement. Journal of Cement and Concrete Resolution. 34(4), 677-680.
Da Cunha, T.B., Wu, J.P., Peitl, O., Fokin, V.M., Zanotto E.D., Lanucci L. ve Boccaccini A.R. 2007. Mechanical properties and impact resistance of a new transparent glass-ceramic. Advanced Engineering Materials, 9(3), 191-196.

Da Silva, R.C., Pianaro, S.A. ve Tebcherani, S.M., 2012. Preparation and characterization of glazes from combinations of different industrial wastes. Ceramics, 38, 2725-2731.

Değirmenci, N., 2008. Utilization of phosphogypsum as raw and calcined material in manufacturing of building products. Construction of Building Materials, 22(8) 1857-1862.

Değirmenci, N., Okucu, A. ve Turabi, A., 2007. Application of phosphogypsum in soil stabilization. Journal of Building and Environment, 42(9), 3393-3398.

Demirel, Y. ve Çağlar, Y., 2015. Atık fosfojipslerin yap1 malzemesi olarak ekonomiye geri. kazandırılması. Gazi Üniversitesi Mühendislik ve Mimarlık Fakültesi Dergisi, 30(4), 743-750.

DIN 53211. 1987. Paints, varnishes and similar coating metarials. Determination of flow time using the flow cup, 1-6. 
El-Didamony, H., Gado, H.S., Awwad, N.S., Fauzy M.M. ve Attallah M.F., 2013. Treatment of phosphogypsum waste produced from phosphate ore processing. Journal of Hazardous Materials, 244-245, 596-602.

Ezz-Eldin, F.M. ve Nageeb, W.M., 2001. Chemical resistance of some irradiated ceramic glasses. Indian Journal of Pure and Applied Physics, 39, 514-524.

Flinders, M., Ray D., Anderson, A. ve Cutler, R.A., 2005. High toughness silicon carbide as armor. Journal of American Ceramic Society, 88(8), 2217-2226.

Formosa, J., Chimenos, J.M., Lacasta, A.M. ve Niubo M., 2012. Interaction between low-grade magnesium oxide and boric acid in chemically bonded phosphate ceramics formulation. Ceramics International, 38(3), 2483-2493.

Garg, M., Minocha, A.K. ve Jain, N., 2011. Environmental hazard mitigation of waste gypsum and chalks: use in construction materials. Construction of Building Materials, 25(2) 944949.

Gennari, R., Garcia, I., Medina, N.H. ve Silveira, M.A.G., 2011. Phosphogypsum Analysis: Total Content and Extractable Element Concentrations, October 2011, Belo Horizonte, Brasil.

Jonker, A. ve Potgieter, J.H., 2005. An evaluation of selected waste resources for utilization in ceramic materials apllications. Journal of European Ceramic Society, 25, 3145-3149.

Li, Y. ve Chen, B., 2013. Factors that affect the properties of magnesium phosphate cement. Journal of Cement and Construction Building Materials, 47(7), 977-983.

Lin, D.F., Lou, H.L. ve Sheen, Y.N. 2005. Glazed tiles manufactured from incinerated sewage ash and clay. Journal of Air and Waste Managment Association, 55, 163-172.

Mc Quillan, F., 1995. The Response of Silceram GlassCeramics to Projectile Impact, $\mathrm{PhD}$ thesis, Imperial Collage of London.

Mejia, J.F., 2004. Understanding the Role of Fluxes in Single-fire Porcelain Glaze Development, MSc Thesis, Alfred University, NY.

Muller, K. ve Zamek, J., 2011. The Potter's Complete Studio Handbook: The Essential Start to Finish Guide for Ceramic Artists, Quarry Books, Beverly Massachusetts.

Parmelee, C.W., 1987. Ceramic Glazes. Cahners Books Division of Cahners Publishing Company, New York.
Singer, F. ve Singer, S.S., 1979. Industrial Ceramics, Chapman and Hall. London.

Singh, M., 2002. Treating waste phosphogypsum for cement and plaster manufacture. Journal of Cement and Concrete Resolution, 32(7), 10331038.

Sirazhiddinov, N.A., Irkakhodzhaeva, A.P. ve Azizkhodzhaeva M.M., 1994. Use of phosphogypsum and floatation wastes from copper concentration for fabrication of glass and glass ceramics. Glass and Ceramics, 51, 102-104.

Smadi, M.M., Haddad, R.H. ve Akour, A.M., 1999. Potential use of phosphogypsum in concrete. Cement and Concrete Research, 9, 1419-1425.

TS EN ISO 10545-13, 2017. Ceramic Tiles-Part 13: Determination of chemical resistance, 1-20.

TS EN ISO 10545-16: 2000. Ceramic Tiles-Part 16: Determination of small colour differences, 1-13.

TS EN ISO 10545-3, 2018. Ceramic Tiles-Part 3: Determination of water absorption, apparent relative density and bulk density, 1-8.

TS EN ISO 10545-5, 2000. Ceramic Tiles-Part 5: Determination of impact resistance by measuring the coefficient of restitution, 1-11.

TS EN ISO 10545-9, 2000. Ceramic Tiles-Part 11: Determination of resistance to thermal shock., 17.

Uhlmann, D.R. ve Keidl, N.J., 1983. Glass Science and Technology Vol:1, New York.

URL-1,

https://www.fipr.statefl.us/publication/phosphogy psum-proceedings-of-the-internationalsymposium-on- phosphogypsum.2007

URL-2, https://www.kalkinma.gov.tr/docobjects/downloa d/3570/oik690.pdf

URL-3, https://gardco.com/pages/viscosity/vi/din_cups.cf m.

URL-4, https://www.hunterlab.com/appnotes/an0896a.pdf

URL-5, https://www.spectrumglazes.com/viscpg.html (accessed 08.06.2018)

Wang, A.J., Yuan, Z.L., Zhang, J., Liu, L.T., Li, J.M. ve Liu, Z., 2013. Effect of raw material ratios on the compressive strength of magnesium potasium phosphate chemically bonded ceramic. Materials Science and Engineering C: Materials for Biological Applications, 38(8), 5058-5063. 
Yalçın, N. ve Sevinç, V., 2000. Utilization of bauxite waste in ceramic glazes. Ceramics International, 26, 485-493.

Zhou, J., Gao, H., Shu, Z. Wang, Y.X. ve Yan, C., 2012. Utilization of waste phosphogypsum to prepare non-fired bricks by a novel hydration- recrystallization process. Journal of Construction and Building Materials, 34, 114-119.

Zhou, J., Sheng, Z., Li T., Shu, Z., Chen, Y. ve Wang C., 2016. Preparation of hardened tiles from waste phosphogypsum by new intermittent pressing hydration. Ceramics International, 42, 7237-7245. 